\title{
Análisis estratégico de grupos de interés e imagen corporativa de las empresas privadas venezolanas
}

\author{
Perozo de Jiménez, Graciela* \\ Del Valle Urdaneta, Marisela**
}

\section{Resumen}

El presente estudio tuvo como propósito determinar las relación existente entre el análisis estratégico de grupos de interés y la imagen de las corporaciones privadas venezolanas. La investigación siguió la modalidad de campo tipo correlacional con un diseño no-experimental - transeccional. La población estuvo constituida por los Gerentes de Planificación e Imagen de las 10 corporaciones privadas venezolanas de mayor tamaño y cuatro expertos académicos en las áreas de Planificación e Imagen Corporativa. Como instrumentos de recolección de datos se utilizaron un cuestionario cerrado de 35 items para ser aplicado a los gerentes de las empresas y una entrevista abierta de 15 preguntas dirigida a los cuatro expertos académicos. Estos se sometieron a la validez de 10 expertos en el área de mercadeo, imagen corporativa, planificación estratégica y metodología. La confiabilidad del cuestionario fue de un coeficiente de 0,90 . El estudio presenta las siguientes conclusiones en función de los resultados obtenidos en la investigación. Los procesos de formulación estratégica de las corporaciones privadas líderes venezolanas están caracterizados por el uso mayoritario de la Planificación Estratégica como herramienta para definir lineamientos estratégicos y guías para la acción en su gestión. En relación a su vinculación con el Análisis de Grupos de Interés, los procesos de Imagen Corporativa son vistos, por los expertos, como el elemento interfase entre los Grupos de Interés y la estrategia global de la corporación.

Palabras clave: Grupos de interés, análisis de grupos de interés, imagen corporativa.

Recibido: 21-07-05. Aceptado: 24-02-06

* Doctora en Ciencias Gerenciales. Profesora Asociado de la Facultad de Ciencias Económicas y Sociales de la Universidad del Zulia (LUZ). E-mail: grajimenez@cantv.net

** Doctora en Ciencias Gerenciales. Profesora de Postgrado Universidad Dr. Rafael Belloso Chacín. E-mail: murdaneta@guasare.com 


\section{Strategic Analysis of Interest Groups and Corporate Image in Private Venezelan Industries}

\section{Abstract}

The purpose of this study was to determine the existing relationships between strategic analysis of interest groups and the image of private Venezuelan corporations. The research mode was a correlational field study with a non-experimental transectional design. The population consisted of planning and image managers from ten private large Venezuelan corporations and four academic experts in the areas of planning and corporate image. Data collection instruments used were a closed questionnaire of 35 items applied to the company managers and an open interview of fifteen questions addressed to the academic experts. These were submitted for validation to ten experts in the areas of merchandising, corporate image, strategic planning and methodology. The questionnaire had a reliability coefficient of 0.90 . The study produced the following conclusions based on results obtained from the research: the strategic formulation processes of leading private Venezuelan corporations are characterized principally by the use of strategic planning as a tool for defining strategic guidelines and guidance for action in their administration. Regarding their connection to the analysis of interest groups, the processes of corporate image are seen by experts as the interface element between the interest groups and the corporation's global strategy.

Key words: Interest groups, analysis of interest groups, corporate image.

\section{Introducción}

En estos tiempos de transformaciones vertiginosas la estrategia de las empresas ha adquirido cada vez mayor importancia. La apertura de los mercados y la aceleración del cambio tecnológico han impulsado la competencia, los riesgos y las recompensas potenciales para las organizaciones plantea que la formulación de estrategias acertadas resulta vital para sobrevivir y prosperar.

Dentro de este marco referencial, en la actualidad y más aún en el futuro, el éxito y la supervivencia de cualquier empresa dependerán significativamente del grado de interacción constructiva con los Grupos de Interés que ejercen influencia sobre las decisiones corporativas.
En tal sentido, Hill y Jones (1996), señalan que los grupos de interés son individuos o conjuntos de personas poseedores de algún derecho sobre la compañía, están constituidos por personas, grupos, instituciones o comunidades capaces de influenciar significativamente el proceso para la formulación e implementación de estrategias, en acuerdo con su interés y poder sobre el quehacer corporativo.

Por su parte Dess y Lumpkin (2003) definen a los grupos de interés como un individuo o grupo, dentro o fuera de la compañía, que tiene interés en ella y puede influir en el desempeño de la organización.

En consecuencia y en base a estas definiciones, la empresa debería incorpo- 
rar esta orientación como parte de su proceso y cultura gerencial, muy especialmente, en los distintos niveles de planificación estratégica cuya finalidad es asegurar el éxito corporativo en la implementación de la Estrategia, dentro de un entorno empresarial signado por la complejidad y dinamicidad. Se puede citar por ejemplo el caso de la reconocida empresa norteamericana Sears, la cual como parte de un proceso de transformación del negocio, desarrolló un modelo cuantitativo para predecir la relación entre la satisfacción de los trabajadores, la de los clientes y los resultados financieros.

Esta empresa descubrió que un $5 \%$ de mejora en la actitud de los empleados conduce a un $1.3 \%$ de mejora en la satisfacción de los clientes, lo cual a su vez lleva a un $0,5 \%$ de mejora de los ingresos" (Rucci, Kim y Quin, 1998: 82-97). Una clara muestra de expectativas de satisfacción de diversos grupos de interés de la empresa en forma complementaria fue producto de un proceso de análisis de sus requerimientos y formas de vincularlos.

En principio, este análisis le permitirá a la empresa entre otras cosas, la identificación de grupos, personas e instituciones influyentes sobre la Estrategia y/o Iniciativa Corporativa, la anticipación de reacciones frente al quehacer corporativo de la empresa, la identificación de los conflictos de interés entre los mismos y el desarrollo de estrategias para apoyar sostenidamente a la estrategia corporativa o en todo caso, reducir los obstáculos que se vislumbren.

Al respecto Lira (2002: 2) señala que "La empresa debería entonces, contar con un proceso metodológico para el análisis de grupos de interés (stakehol- ders) el cual ordenada y sistemáticamente facilite determinar el posicionamiento de esos grupos con relación a las estrategias e iniciativas de la Corporación".

Asimismo, Hill y Jones (1996:46) refieren que "una compañía no siempre puede satisfacer las exigencias de todos los grupos de interés. Los derechos de diferentes conjuntos de individuos pueden generar conflicto, y en la práctica pocas organizaciones cuentan con los recursos para manejar los grupos de interés".

Se evidencia en la cita de estos autores el impacto que estos grupos pueden tener en la gestión de las organizaciones versus los recursos con los que se cuenta para satisfacer sus requerimientos. En consecuencia, es importante considerar la aplicación de una metodología que permita su identificación y jerarquización a fin de formular estrategias dentro del Plan de Negocios que contemplen su manejo.

Cabe destacar como las presiones de los Grupos de Interés son de naturaleza diversa; las mismas abarcan desde la agregación de valor corporativo hasta demandas sociales en términos de empleo, servicios e inversión social. Sin embargo, para las corporaciones venezolanas las presiones fiscales recurrentes, las crecientes exigencias de las comunidades y sociedad general en términos de generación de empleo, contribuciones sociales e integración intersectorial constituyen los requerimientos mas demandados por estos grupos.

También concurren presiones por parte de públicos objetivos de los productos y mensajes de las corporaciones, mayores exigencias en relación a la calidad en una economía en franco deterioro, re- 
gulaciones gubernamentales de todo orden y activistas ambientales, entre otros.

El movimiento de estos grupos impacta significativamente los distintos niveles de gestión en la Corporación: operacional, negocio y táctico estratégico e inclusive la propia gobernabilidad y mecanismos para el control de la gestión empresarial. La influencia de estos, llega entonces a la base de la Cultura Corporativa que fundamenta la forma como la empresa desea ser percibida por sus públicos internos y externos.

Por su parte, la Cultura Corporativa es, el elemento que le da sentido a la vida organizacional. Dicha cultura se desarrolla dentro de los referentes interpretativos que tienen los miembros en la organización para darle significado a las acciones cotidianas de la empresa. ¿Cómo se forman estos referentes? A través de los distintos actos comunicacionales, que apuntan a una simbología compartida por todos sus miembros o grupos de interés.

Asimismo, los medios de información son el camino por el cual transitan esos actos comunicacionales; es decir, los mensajes de la corporación y ellos nacen de un sistema cultural formado por la empresa a través de los años. Asesores de Imagen e Identidad Corporativa, plantean que la cultura o forma de ser y hacer empresa, es continuamente afectada por los cambios del entorno y sus nuevas exigencias; sin embargo, cuando se diseña la identidad o personalidad construida por la empresa y demás rasgos característicos, son muchos los sesgos que la marcan frente al futuro y no siempre con resultados positivos (Pizzolante, 1999a).

Como consecuencia de ello, las organizaciones que se involucran en un proceso para la transmisión de mensajes a fin de construir una imagen positiva basada en la reputación de la empresa, han salido favorecidas claramente en el análisis crítico de sus estructuras interna y externa, así como estar conscientes de las fortalezas y debilidades, amenazas y oportunidades factibles de impactar su Plan de Negocios.

En esta investigación el interés se centró en estudiar la relación entre el análisis de grupos de interés (stakeholders) como parte del proceso de formulación estratégica en la corporación con el diseño de estrategias para la proyección de la imagen de estas empresas en su entorno.

Asimismo, se busco establecer relaciones, herramientas y lineamientos para una mejor comprensión y utilización de estos dos procesos gerenciales; es decir, Planificación Estratégica e Imagen Corporativa enfocados hacia una visión integradora que tome en cuenta las vinculaciones más importantes entre ambos. El aporte de este estudio, consistió en analizar profundamente aspectos de la estrategia de negocios que hasta ahora han sido vistos aisladamente o poco relacionados entre si como son la Planificación Estratégica del Negocio y la Imagen Corporativa. Por lo tanto la motivación del presente artículo estuvo enmarcado en el objetivo de: Determinar las relaciones existentes entre el análisis estratégico de los grupos de interés y la imagen de las corporaciones privadas venezolanas con mayor tamaño y líderes en el país (Frances 2001).

En este sentido, el estudio se inserta en las investigaciones correlaciónales, según Salkind (1999), este tipo de estudio describe la relación lineal entre dos o 
más variables sin tratar en lo absoluto de atribuir a una variable el efecto observado en otra.

La investigación siguió modalidad de campo con un diseño no-experimental - transeccional. La población estuvo constituida por los Gerentes de Planificación e Imagen de las 10 corporaciones privadas venezolanas de mayor tamaño existentes en el país, consideradas por Francés (2001) corporaciones líderes del año 2000, las cuales manejan de forma organizada y estructural los procesos de planificación estratégica y formulación de estrategias de imagen corporativa como parte de su gestión empresarial, entre las que consideró: En el Sector Industrial: Polar (alimentos y bebidas), Corpalmar (azúcar, alfarería, embutidos), Sivensa (metalurgia y metalmecánica), Sector Financiero: Grupo Banco Mercantil (banca), Sector Servicios: Grupo 1BC (radio y televisión), Organización Diego Cisneros (medios masivos y telecomunicaciones), Otepi (servicios de ingeniería), Inelectra (servicios de ingeniería), Cantv (telecomunicaciones), La Electricidad de Caracas (servicio eléctrico) y cuatro expertos académicos, en las áreas de Análisis Estratégico de Grupos de Interés e Imagen Corporativa del Instituto de Estudios Superiores de Administración (IESA), Universidad del Zulia (LUZ) y Universidad Rafael Belloso Chacín (URBE) Como instrumentos de recolección de datos se utilizaron un cuestionario cerrado de 35 items para ser aplicado a los gerentes de las empresas y una entrevista abierta de 15 preguntas dirigido a los cuatro expertos académicos. Estos se sometieron a la validez de 10 expertos. La con- fiabilidad del cuestionario obtuvo un coeficiente de 0,90.

\section{La necesidad de un nuevo enfoque de análisis de la Imagen Corporativa}

La imagen no debe pensarse, como una máscara externa que puede adaptarse convenientemente a las situaciones de la organización, por el contrario, la imagen debe ser una prolongación o proyección de la estructura y comportamiento de la empresa en la cual conviven los stakeholders del negocio. Pero ¿qué es la imagen corporativa y sobre todo cuál es su importancia en el éxito o fracaso de los negocios? Para dar respuesta se podría expresar que la imagen corporativa es un acto deliberado en doble sentido: por ser voluntario y por suponer una formulación. El autor Pizzolante (1999: 3) señala: Los principios, normas y valores de la empresa, su trayectoria y el comportamiento de su personal, modelan lo que se ha llamado la Cultura Corporativa, forma de ser y hacer empresa que constituye la Identidad Corporativa y que al ser proyectada crea el fenómeno de opinión pública denominado imagen

Por esta razón, la imagen corporativa no puede ser producto del azar o de la suerte, sino producto de un acto razonado que conlleve al éxito de a empresa. En este sentido, Madia De Souza (1995), plantea que durante los años 80 , los ejecutivos comenzaron a contemplar el mercado y meditar sobre él. A través de esta experiencia realizaron algunos descubrimientos, de los cuales el mayor y más importante fue el reconocimiento vinculado a la existencia, dentro de ese mercado, 
de un valor empresarial sobresaliente en importancia sobre todos los demás valores inherentes a ella: la imagen percibida por las personas involucradas a sus procesos decisorios.

El referido autor confirma lo planteado por Pizzolante (1999), en lo relativo a la importancia de tener una adecuada estructura corporativa interna a fin de proyectar sistemáticamente comportamientos y comunicaciones promotores de una imagen positiva de la empresa. A medida que éstas se conocen internamente, partiendo del registro del sentimiento de sus públicos decisivos y vitales, descubren su necesidad de organizar mejor los elementos inherentes a su cultura corporativa antes de exponerse.

Por una parte, vincular ese enfoque interno con el uso de un proceso estructurado que permita la identificación y posicionamiento, es decir intereses y expectativas de los grupos influyentes en la estrategia organizacional, posibilitará formular opciones estratégicas para imagen corporativa las cuales beneficiarán su gestión global.

En este sentido, para toda empresa es importante recordar que no basta un buen desempeño, debe contar también con una buena imagen corporativa como garante del impacto factible de alcanzar en su contexto: una empresa sin imagen conlleva a no ser considerada por su público, es decir, a una situación donde nadie piensa en ella, lo cual, sin lugar a dudas, impactará negativamente los resultados del negocio.

Por otra parte, la imagen corporativa de una empresa debe ser producto de una secuencia ordenada e inteligente de eventos constructivos, con visión a largo plazo. La idea básica será construir un perfil público basado en el prestigio y la reputación que posea la organización como elementos permanentes.

\section{La Identidad Corporativa en las organizaciones}

La identidad corporativa, se define como la personalidad construida por la empresa. Esta no es una cualidad innata en la organización, sino el resultado de un esfuerzo consistente por descubrir las fortalezas, debilidades y potencialidades mediante operaciones de auto evaluación y definición de la singularidad empresarial. Al conocerse, entonces, el clima organizacional y a partir de allí concluir sobre las fortalezas y debilidades en la cultura, es la forma más eficaz de conocer y afectar positivamente la identidad de una empresa (Pizzolante, 1999).

La fase siguiente es construir, a partir de esa identidad, la imagen corporativa de la organización para que, en el mediano y largo plazo, sea un proceso continuo de proyección desde adentro hacia fuera.

En tal sentido, el desarrollo de los mensajes que construirán la imagen debe tomar en cuenta los componentes de la cultura empresarial y los públicos a ser influenciados por estos mensajes.

Tal reflexión impulsa a indagar sobre cuál es la situación en las empresas venezolanas y cómo manejan sus relaciones con los grupos que pueden impactar la estrategia de la organización. 


\section{La cultura organizacional en Venezuela}

En el caso venezolano, destaca la cultura del bajo perfil, es decir, una cultura fundamentada sobre una relación débil con el entorno donde desarrolla sus actividades, pudiéndose mencionar, vecinos, medios de comunicación, políticos, gobierno. Estas empresas generalmente han desarrollado una sólida relación con el mundo interior, tal como vocación al logro, superación personal, formación a la excelencia, altos beneficios para el personal pero sin exponer o mostrar los efectos positivos de esta cultura a los factores que movilizan su ambiente externo de decisiones, lo que impide el aprovechamiento de estas bondades (Pizzolante, 1999: 68).

Según Pizzolante, 1999, estas empresas crearon una generación de empleados con altos niveles de seguridad, muy preparados profesionalmente, pero "inefectivos a la hora de las huelgas, los titulares de prensa adversos, las manipulaciones políticas y las presiones del gobierno".

Además, "estas organizaciones formaron ejecutivos con patrones conductuales caracterizados por trabajar con muy buena intención, tomando o dejando de tomar decisiones sin ver los riesgos vinculados a sus actuaciones", lo cual genera que en la rendición de cuentas de la acción gerencial priva la filosofía de que, en general, la empresa no mide sus esfuerzos mas allá de las unidades para la producción de bienes, de la rentabilidad, el volumen de mercado y las ganancias obtenidas.

Esta realidad descrita por el referido autor plantea que el reto gerencial de las empresas privadas venezolanas es repensar a la organización para insertarla en las nuevas realidades sociales y políticas. Para ello es necesario modificar su cultura y posteriormente su identidade imagen como pasos previos y vitales. No basta con estar bien preparados académicamente, tener productos competitivos y hasta prestigio empresarial construido, es importante hacer seguimiento proactivo a los cambios del entorno midiendo continuamente las implicaciones en los negocios, a fin de establecer a tiempo los correctivos del caso.

\section{La proyección de la imagen corporativa en las empresas Venezolanas}

Con relación a los procesos de proyección de la imagen corporativa, la situación en las empresas privadas venezolanas se pueden observar dos vertientes. En la primera, las organizaciones no cultivan procesos organizados para la proyección de su imagen corporativa, y en ocasiones ni siquiera han construido la identidad corporativa en sus organizaciones. Este es al parecer el caso clásico de un significativo porcentaje de las pequeñas y medianas empresas o industrias privadas del país.

La imagen corporativa en las corporaciones o empresas grandes de capital privado venezolano, es básicamente un proceso manejado por los departamentos de Relaciones Públicas, Asuntos Públicos o Mercadeo. Dichos departamentos orientan sus funciones y actividades hacia los públicos externos de la organización, lo cual es válido. Sin embargo, en un entorno tan dinámico como el 
actual donde las organizaciones que han llegado a incursionar al siglo XXI deben reflexionar sobre su imagen corporativa como el mayor de los patrimonios de las empresas, se puede afirmar que la construcción de ésta debe ser un objetivo fundamental del Plan de Negocios de las organizaciones tal como lo señala Madia Di Souza (1995).

\section{El Proceso de Formulación de estrategias de Imagen y los grupos de interés}

Ante este planteamiento cabe reflexionar lo siguiente: el proceso de formulación de estrategias de imagen vinculadas al plan de negocios pasa por miradas profundas hacia dentro y fuera de la organización, a través de la cual encontran los grupos de interés con su visión y expectativas sobre la corporación. El análisis sistemático de estos grupos de interés es de especial relevancia para la consecución del éxito corporativo, en términos de supervivencia y crecimiento a mediano y largo plazo. Sin embargo, la gran pregunta es ¿cómo hacerlo? y ¿Cómo impacta el análisis de los stakeholders del negocio los procesos para formular estrategias de imagen corporativa? Para dar respuesta a la misma, según la visión de Pizzolante (1996:22):

No todo individuo o empresa está en condiciones directas e inmediatas de construir una estructura corporativa que le otorgue reputación (imagen) más allá de la calidad del producto y capacidad operativa (eficiencia). La condición previa para ello es haber salido favorecido claramente en el análisis crítico cualitativo de su estructura interna y adicionalmente estar conscientes de las amenazas y oportunidades que condicionan el Plan de Negocios de la empresa.

Se visualiza una potencial conexión entre la necesidad de un enfoque metodológico que haga seguimiento a las expectativas de todos los públicos que percibirán la imagen de la organización. Estos públicos pueden ser asociados al concepto de Grupos de Interés de las organizaciones, ampliando significativamente el concepto de públicos internos y externos que se maneja actualmente en la teoría de imagen corporativa.

Tal relación aportará herramientas que permitirán profundizar los análisis de quiénes a través de sus percepciones y expectativas impactan las estrategias de las empresas. "Iniciar un programa de Imagen sin estrategia es como despegar sin haber preparado un plan de vuelo, ni considerar los informes meteorológicos del día" (Pizzolante, 1996: 80).

\section{Los resultados del estudio}

Se presenta a continuación el resultado del valor obtenido para expresar el grado de correlación existente entre las variables Análisis Estratégico de Grupos de Interés y Proceso de Formulación de Imagen Corporativa. El valor obtenido fue de 0.56 lo cual indica una correlación moderada entre ambas variables (Tabla 1).

\section{Tabla 1}

\section{Correlación de Pearson}

0.5594302

Fuente: Elaboración propia (2004). 
Al revisar los datos obtenidos producto del cuestionario aplicado a los Gerentes de Planificación e Imagen y a las entrevistas dirigidas a los expertos en estas áreas, se pudo observar como resultado los siguientes aspectos: En cuanto al Análisis Estratégico de Grupos de Interés en la dimensión: Proceso de Formulación Estratégica, ambas poblaciones, es decir, los Gerentes de Planificación y los de Imagen Corporativa afirman que las corporaciones utilizan la Planificación Estratégica como herramienta para visualizar el futuro de la organización, Esta visión, aun cuando prevalece en ambos grupos, tiene algunas variaciones que vale la pena destacar. En la población "Gerentes de Planificación" se menciona la Planificación Táctica como la segunda de sus opciones, mientras que los "Gerentes de Imagen" conciben la Planificación de Negocios como la segunda alternativa.

En cuanto a la Coordinación del Proceso, los Gerentes de Planificación otorgan más peso al sector que representan con una importante participación de la Presidencia de la empresa. Los Gerentes de Imagen también concentran sus menciones en ambas alternativas, pero igualando el peso de ambas opciones. Esta interpretación se valida cuando ambas poblaciones confirman el rol de coordinación en el Proceso de Formulación de Planes a la Gerencia de Planificación.

En este sentido, los expertos académicos entrevistados muestran una alta tendencia a mencionar la Planificación de Negocios como la más utilizada por las corporaciones privadas, indicando la necesidad de avanzar hacia un horizonte de planificación a más largo plazo tal como lo plantean Frances (2001), Steiner (1969), Hill y Jones (1996) y Cuplit, Center y Broom (2001). También señalan la oportunidad de moverse hacia la implantación de Comités Multidisciplinarios dirigidos por la "Alta Dirección" a fin de integrar visiones múltiples en el proceso de Formulación Estratégica como elemento clave para garantizar el futuro.

Por otra parte, destacaron el nivel de involucramiento que deben tener todos los departamentos en la formulación estratégica lo cual se alinea con Dess y Lumpkin (2003) quienes señalan que las organizaciones no son eficaces si la alta dirección realiza la reflexión y el resto de la organización ejecuta el trabajo; es decir, que todos los niveles decisorios deben estar involucrados en el proceso de dirección estratégica.

Con relación al rol de la Gerencia de Planificación dentro de este proceso, las tendencias se ubican entre Coordinador del Proceso con un alto $89.2 \%$ y de Asesor del proceso con $10.8 \%$., lo cual es consistente con los resultados mostrados en la Tabla 2, sobre la coordinación de este importante proceso para visualizar el futuro de la empresa. La Tabla 2, sintetiza estos resultados.

En relación a la Dimensión: Utilización del Análisis Estratégico, dentro del proceso de formulación estratégica la definición de la misión es, según Hill y Jones (1996) el principal indicador de cómo la organización visualiza las exigencias de los grupos de interés. Con relación a ésta área, los gerentes y expertos informaron que la revisión de la misión es un proceso que se efectúa esporádicamente, de lo cual pudiera inferirse que las expectativas de los grupos no son constantemente 


\section{Tabla 2 \\ Tipo de participación de la gerencia en el proceso de formulación de planes}

\begin{tabular}{lcc}
\hline \multicolumn{1}{c}{ ALTERNATIVAS } & FA & FR \\
\hline Líder del Proceso & 0 & $0.0 \%$ \\
Coordinador del Proceso & 9 & $89.2 \%$ \\
Asesor del Proceso & 1 & $10.8 \%$ \\
Control de Indicadores & 0 & $0.0 \%$ \\
Otro & 0 & $0.0 \%$ \\
TOTAL & 10 & $100.0 \%$ \\
\hline
\end{tabular}

Fuente: Elaboración propia (2004).

revisadas con relación a la exposición de la misión, elemento estratégico que vincula las expectativas de los stakeholders a las organizaciones. Contrasta este resultado con el alto impacto que ambas poblaciones otorgan a los Grupos de Interés en la Formulación e Implantación de las Estrategias del Negocio.

En términos del uso del "Análisis Estratégico de Grupos de Interés", ambas poblaciones indican a través de su selección de alternativas, un uso moderado de la herramienta aun cuando la consideran Muy Adecuada para la Formulación Estratégica, tal como lo recomienda el modelo de Arthur D'Little presentado por Kottler (1996) donde los Grupos de Interés y sus necesidades son vistos como el punto de partida de la formulación estratégica de cualquier compañía. Sin embargo, se plantean la formulación de estrategias para el manejo de grupos de interés en ambos casos con un alto porcentaje de forma anual, lo cual pudiera tener que ver, como lo señalan los expertos, con el manejo de sus grandes situaciones de negocio en la planificación a corto plazo.
En este punto los expertos académicos entrevistados coinciden en la definición de la misión como un proceso compartido que debe consustanciar los grupos de interés con el horizonte de la organización. No puede ser impositiva bajando desde la gerencia en procesos de consulta frágiles basados en el poder centralizado. Con relación a la participación actual de los stakeholders o actores claves, aclaran que actualmente su participación es marginal y la tendencia debe ser a avanzar hacia la oportunidad de comunicarse y negociar con ellos, al menos lo más impactantes, para lograr una misión consensuada.

En referencia al uso del Análisis Estratégico de Grupos de Interés en las corporaciones venezolanas, señalan que se están haciendo algunos esfuerzos, pero que no es tan frecuente como los tiempos modernos exigen. La consolidación de alianzas producto de estas acciones analíticas pudiera medir cuan avanzado está el pensamiento estratégico en las empresas venezolanas.

Se aprecia que la Gerencia de Imagen Corporativa tiene un alto nivel de in- 
volucramiento en el proceso de formulación estratégica, aspecto coincidente en la selección de ambas poblaciones y confirmado desde el punto de vista teórico por Pizzolante (1999b) quien señala que la imagen se sustenta en la Comunicación Estratégica, Identidad y Cultura Corporativa como estrategia corporativa de la confianza orientada al cumplimiento del Plan de Negocios. En opinión de la población de expertos (del área de Imagen) todo lo que gira en torno a la comunicación es medular del negocio. La imagen tiene impacto determinante en la concepción y desarrollo de negocios.

Consideran ambas poblaciones que la gestión de los grupos de interés (stakeholders) tiene un alto impacto en la formulación de estrategias de Imagen Corporativa y que el uso del Análisis Estratégico de Grupos de Interés es una herramienta útil a la hora de formular estas estrategias.

Vale la pena destacar que en este sentido los expertos consideraron a la Imagen Corporativa como el elemento vinculador e interpretador de las expectativas de los Grupos de Interés hacia la formulación estratégica global de la corporación. La Imagen Corporativa es la inter- fase del fin que es la estrategia y determina el impacto de las necesidades y expectativas de los stakeholders en la estrategia global de la corporación.

El indicador "Estrategias de Grupo de Interés" orientado a medir utilización del enfoque en el proceso de planificación estratégica, muestra a través de la Tabla 3, que su utilización es alta al concentrarse el $60 \%$ de las respuestas en las categorías Frecuentemente, Anualmente y Cada Dos Años, desde la perspectiva de esta población. Cuatro de las empresas (40\%) indicaron utilizar el análisis Esporádicamente.

Esto es consistente con los resultados mostrados en la Tabla 4, en la cual $60 \%$ de las respuestas se orientaron a las categorías Muy Adecuado e Indispensable en términos el uso de esta herramienta en la formulación estratégica; seguido muy de cerca $(30 \%)$ por la categoría Adecuado y Medianamente Adecuado (10\%). Ninguna de las empresas respondió que consideraba Inadecuado el Análisis Estratégico de Grupos de Interés.

En lo relativo a la dimensión: Estrategias de Comunicación, partiendo de la definición de Pizzolante (1996) de la imagen como una estructura mental apunta-

\section{Tabla 3 \\ Utilización del análisis estratégico de grupos de interés por las empresas}

\section{ALTERNATIVAS} Frecuentemente Anualmente Cada dos Esporádicamente Nunca TOTAL
años

\begin{tabular}{ccccccc}
\hline FA & 2 & 2 & 2 & 4 & 0 & 10 \\
FR & $20 \%$ & $20 \%$ & $20 \%$ & $40 \%$ & $0 \%$ & $100 \%$ \\
\hline
\end{tabular}

Fuente: Elaboración propia (2004). 


\section{Tabla 4}

\section{El análisis estratégico de grupos de interés en la formulación de estrategias de negocio}

\begin{tabular}{ccccccc}
\hline & \multicolumn{5}{c}{ ALTERNATIVAS } & \\
Indispensable & $\begin{array}{c}\text { Muy } \\
\text { adecuado }\end{array}$ & Adecuado & $\begin{array}{c}\text { Medianamente } \\
\text { adecuado }\end{array}$ & Inadecuado & TOTAL \\
\hline FA & 2 & 4 & 3 & 1 & 0 & 10 \\
FR & $20 \%$ & $40 \%$ & $30 \%$ & $10 \%$ & $0 \%$ & $100 \%$ \\
\hline
\end{tabular}

Fuente: Elaboración propia (2004).

lada en los elementos de la cultura organizacional y la identidad corporativa proyectada a través de la comunicación para convertirse en Imagen Corporativa, se evaluó el proceso de formulación estratégica asociadas a cada uno de estos componentes y su correspondiente vinculación con la otra variable de estudio Análisis Estratégico de Grupos de Interés.

En términos de Estrategias de Comunicación se determinó que el volumen de información y que el nivel de comunicación formal dentro de las grandes corporaciones es Alto así como los volúmenes de información y nivel de uso de la Comunicación Corporativa como medio de transmisión de esa información hacia sus stakeholders, lo cual fue validado por ambas poblaciones.

El Grupo de Interés principal para ambos grupos son los clientes, seguidos por los accionistas y empleados de la corporación. En el caso de los Líderes de Planificación le sigue en importancia las instituciones financieras, mientras que los Líderes de Imagen Corporativa continúan con el Gobierno como principal público.

Como se visualiza, en referencia a los Públicos Objetivo o Grupos de Interés de las Corporaciones (Tabla 5), los Gerentes de Planificación confirmaron que los Clientes son sus principales públicos (20.4\%), seguidos muy de cerca por los Accionistas y Empleados (ambos con $18.4 \%$ ) y las Instituciones Financieras (14.3\%). Un 12.2\% incorporó al Gobierno como uno de sus públicos. Los Sindicalistas $(6.1 \%)$, Inversionistas y Entes Reguladores $(4.1 \%)$ y Comunidad $(2 \%)$ completan estos resultados.

La toma de decisiones en el diseño de estrategias comunicacionales se concentra en la Junta Directiva y la Gerencia de Imagen Corporativa y consideran que el diseño de las mismas es parte del Plan de Negocios de la Corporación.

Ambos grupos consideran la influencia de los stakeholders en el diseño de estrategias de comunicación como alta al igual que el uso del Análisis Estratégico de Grupos de Interés para la formulación de Estrategias de Comunicación.

Por su parte, todos los expertos coincidieron en la importancia que la opinión de los stakeholders tiene para alimentar las políticas comunicacionales de las corporaciones.

Se estableció acá la vinculación entre el uso del Análisis Estratégico de 


\section{Tabla 5}

Principales grupos de interés de la corporación

\begin{tabular}{lcc}
\hline ALTERNATIVAS & FA & FR \\
\hline Clientes & 10 & $20.4 \%$ \\
Accionistas & 9 & $18.4 \%$ \\
Inversionistas & 2 & $4.1 \%$ \\
Empleados & 9 & $18.4 \%$ \\
Sindicalistas & 3 & $6.1 \%$ \\
ONG's & 0 & $0.0 \%$ \\
Familiares & 0 & $0.0 \%$ \\
Instituciones Financieras & 7 & $14.3 \%$ \\
Gobierno & 6 & $12.2 \%$ \\
Entes Reguladores & 2 & $4.1 \%$ \\
Comunidad & 1 & $2.0 \%$ \\
TOTAL & 49 & $100.0 \%$ \\
\hline
\end{tabular}

Fuente: Elaboración propia (2004).

Grupos de Interés y el Proceso de Formulación de Estrategias Comunicacionales confirmándose que un $50 \%$ lo considera Indispensable y el otros $50 \%$ se dividió entre Muy Adecuado y Adecuado, con lo cual se confirma la existencia de una relación entre ambos procesos, tal como se puede apreciar en la Tabla 6.

Tomando en consideración la dimensión: Cultura Organizacional. Pizzolante (1999 b) la define como aquellas informaciones 0 rasgos que no son tangibles ya que se refieren a la filosofía de su vida corporativa: valores, normas y principios. El autor la define como el inconsciente colectivo que se manifiesta explícitamente en la empresa mediante un conjunto de comportamientos.

En este sentido, es importante observar que ambas poblaciones de las corporaciones la definieron como un componente importante de su proceso de plani- ficación estratégica con un Alto nivel de influencia de la misión, visión principios y valores, destacándose éste último en las menciones. Esta información fue validada por la percepción de los expertos.

Con relación al impacto de los Grupos de Interés en la Cultura Organizacional, tanto los Gerentes de Planificación como los de Imagen, consideraron que este es Alto, lo cual se sustenta con la teoría de Ribes (2003) en este sentido. Los expertos en su mayoría validaron esta afirmación hasta el punto de que sugieren el establecimiento de un diálogo comunicacional que permita establecer un nuevo marco de competencias para manejar esta influencia.

En referencia al impacto de las actuaciones de los Grupos de Interés en la cultura, se muestra en la Tabla 7 como un $60 \%$ lo valora como Alto, mientras que el otro $40 \%$ se distribuye en $20 \%$ en el rango 


\section{Tabla 6}

\section{El análisis estratégico de grupos de interés a la hora de formular estrategias de comunicación corporativa}

\begin{tabular}{ccccccc}
\hline \multicolumn{7}{c}{ ALTERNATIVAS } \\
\hline Indispensable & $\begin{array}{c}\text { Muy } \\
\text { adecuado }\end{array}$ & Adecuado & $\begin{array}{c}\text { Medianamente } \\
\text { adecuado }\end{array}$ & Inadecuado & TOTAL \\
\hline FA & 5 & 3 & 2 & 0 & 0 & 10 \\
FR & $50 \%$ & $30 \%$ & $20 \%$ & $0 \%$ & $0 \%$ & $100 \%$ \\
\hline
\end{tabular}

Fuente: Elaboración propia (2004).

Tabla 7

Impacto de las actuaciones de los grupos de interés del negocio en la cultura organizacional

\begin{tabular}{ccccccc}
\hline \multicolumn{7}{c}{ ALTERnATIVAS } \\
\hline Muy Alto & Alto & Medio & Bajo & Muy Bajo & TOTAL \\
\hline FA & 2 & 6 & 2 & 0 & 0 & 10 \\
FR & $20 \%$ & $60 \%$ & $20 \%$ & $0 \%$ & $0 \%$ & $100 \%$ \\
\hline
\end{tabular}

Fuente: Elaboración propia (2004).

inmediatamente superior (Muy Alto) y $20 \%$ en el inmediatamente inferior (Medio). Ninguno respondió en los niveles extremos inferiores (Bajo y Muy Bajo).

Tomando en consideración los aspectos relacionados con la dimensión: Identidad Corporativa. También este pilar fundamental de la Imagen Corporativa fue incluido por los Líderes Planificación e Imagen como parte integral del proceso de formulación estratégico de las corporaciones aunque con menor peso que sus otros dos componentes. Indicaron un alto impacto de los stakeholders en la identidad.

En este sentido Pizzolante (1996) señala la importancia de la Identidad Corporativa como una dimensión estratégica que debe estar vinculada al Plan de $\mathrm{Ne}$ gocios y permite gerenciar la confianza y la reputación como elementos claves de la imagen de una corporación.

Con relación a los elementos que las corporaciones utilizan para proyectar Confianza a sus Grupos de Interés en la Tabla 8 se muestra como la mayoría de las empresas $(38,4 \%)$ utiliza la Solidez Financiera como atributo para generar Confianza en sus stakeholders. Las otras opciones recibieron un $15,4 \%$ cada una.

\section{Conclusiones}

El estudio presenta las siguientes conclusiones en función de los resultados obtenidos en la investigación.

Los procesos de formulación estratégica de las corporaciones privadas líderes venezolanas están caracterizados 


\section{Tabla 8}

\section{La empresa genera confianza en sus grupos de interés}

\begin{tabular}{lcc}
\hline \multicolumn{1}{c}{ ALTERNATIVAS } & FA & FR \\
\hline Su Solidez Financiera & 5 & $38.4 \%$ \\
Programas de Imagen Corporativa & 2 & $15.4 \%$ \\
Programas de Comunicaciones & 2 & $15.4 \%$ \\
Programas de Relaciones con Grupos Claves & 2 & $15.4 \%$ \\
Participación en Instituciones Relacionadas & 2 & $15.4 \%$ \\
TOTAL & 10 & $100.0 \%$ \\
\hline
\end{tabular}

Fuente: Elaboración propia (2004).

por el uso mayoritario de la Planificación Estratégica como herramienta para definir lineamientos estratégicos y guías para la acción en su gestión. El proceso es coordinado por la Gerencia o Departamento de Planificación con alta participación de la Presidencia o Junta Directiva de la empresa en su desarrollo.

Del análisis del instrumento aplicado a los expertos se concluye que la tendencia sería fortalecer apuntando al manejo de los procesos de formulación estratégica a través de Comités Interdisciplinarios donde estén representadas todas las áreas de la corporación.

Con relación a la utilización del Análisis Estratégico de Grupos de Interés se estableció un uso moderado dentro del proceso de formulación estratégica, aunque ambas poblaciones corporativas abordadas otorgaron un alto impacto a la influencia de los stakeholders en la viabilidad de las estrategias de la empresa.

La revisión de la misión, elemento clave para la visualización de las exigencias de los Grupos de Interés, se realiza esporádicamente; sin embargo, la formulación estratégica para el manejo de estos grupos se hace preponderantemente en forma anual, lo cual pudiera tener que ver con el manejo de grandes issues en la planificación a corto plazo. De las opiniones de la población de los expertos, se desprende como la participación activa de los Grupos de Interés es marginal en la formulación estratégica de las empresas.

Las Gerencias de Imagen Corporativa tienen un alto nivel de involucramiento en el proceso de formulación estratégica de las corporaciones y según los expertos, debe tener un impacto determinante en la concepción y desarrollo de los negocios. En relación a su vinculación con el Análisis de Grupos de Interés, los procesos de Imagen Corporativa son vistos, por los expertos, como el elemento interfase entre los Grupos de Interés y la estrategia global de la corporación.

En lo relativo a las Estrategias de Comunicación de las corporaciones privadas venezolanas se determinó que el volumen de información y el nivel de comunicación formal hacia los stakeholders es alto, validándose el uso de la comunicación corporativa como medio de transmisión de esa información a los Grupos de Interés. Para las corporaciones, los stakeholders tienen alta influencia en el 
diseño de estrategias de comunicación al igual que el uso de Análisis Estratégico de Grupos de Interés para la formulación de estrategias en esta área del negocio.

El Grupo de Interés principal para las corporaciones son los clientes, seguidos de los accionistas y empleados de la corporación.

La toma de decisiones en torno al diseño de estrategias comunicacionales se concentra en la Junta Directiva y la Gerencia de Imagen Corporativa.

La cultura organizacional de las corporaciones presenta los siguientes aspectos concluyentes: Fue definida como un componente importante del proceso de planificación estratégica con un alto nivel de influencia de la misión, visión, principios y, especialmente, valores. Con relación al impacto de los Grupos de Interés en la cultura organizacional, los gerentes la calificaron en términos generales como de Alto Impacto, pudiendo inferirse que éstos deberían ser tomados en cuenta en los procesos deliberados de formación de la cultura de las corporaciones. También los expertos le asignaron Alto Impacto, especialmente a los stakeholders internos.

La Identidad Corporativa fue incluida por los Líderes Planificación e Imagen como parte integral del proceso de formulación estratégica de las corporaciones aunque con menor peso que sus otros dos componentes. Indicaron un alto impacto de los stakeholders en la identidad.

Como elementos generadores de confianza a través de su identidad, se señaló la solidez financiera, destacándose con relación a las otras alternativas planteadas. Otras menciones de interés fueron los programas de comunicación y de relaciones con grupos claves por parte de la población "Gerentes de Imagen" los cuales dieron los incorporaron como parte de sus programas para generar confianza en sus stakeholders.

La relación existente entre las variables de estudio, Análisis Estratégico de Grupos de Interés y Formulación Estratégica de Imagen Corporativa se estableció como Moderada - Positiva.

Partiendo de los resultados, surgieron ideas e implicaciones para investigaciones posteriores que amplíen la información sobre la relación entre el Análisis Estratégico de los Grupos de Interés y el Proceso de Formulación de Estrategias de Imagen Corporativa.

Se plantea a las empresas, profundizar en sus procesos de escucha activa de los Grupos de Interés (internos y externos), a fin de anticipar su potencial impacto en los procesos de formulación e implantación de estrategias globales. Los líderes de los procesos de Imagen Corporativa, tienen un rol muy activo al servir como entes de recopilación, interpretación y análisis de las necesidades y requerimientos de los stakeholders claves para el negocio, lo cual debe ser realizado de forma muy cercana a las Gerencias de Planificación de las empresas para así garantizar alineamiento en torno a esta práctica.

Es importante generar esfuerzos y compromisos en la difusión y puesta en práctica de modelos, como el Análisis Estratégico de Grupos de Interés, los cuales permitan a la organización tener disponible la información sobre expectativas, recursos y poder de sus Grupos de Interés, a fin de convertirla en una herramienta que le permita visuali- 
zar potenciales impactos en la estrategia de la empresa. Es importante el desarrollo de una cultura de proactividad y alto sentido político estratégico de quienes tengan a su cargo el proceso. Esta cultura debe ser transferida a todos los miembros de la organización, incluyendo a los tomadores de decisiones estratégicas, diseñando un programa y recursos para este fin.

\section{Referencias Bibliográficas}

Bolman, Lee y Terence, Deal. (2002). Organización y Liderazgo. EUA, Editorial Addison - Wesley Iberoamericana.

Costa, Joan. (2000). Identidad Corporativa. México. Editorial Trillas.

Cutlip, Scott, Center, Allen y Broom, Glenn (2001). Relaciones Públicas Eficaces. Barcelona, España. Editorial Gestión 2000, S.A.

Dess, Gregory y Lumpkin, GT. (2003). Dirección Estratégica: Creando Ventajas Competitivas. España: Editorial McGraw-Hill.

Drucker, Peter. (1954). The Practice of Management. New Cork, EE.UU: Harper \& Row.

Forbes, Paul (1992). Artículo: Applying Strategic Management to Public Relations. Journal 48, № 3 .

Fortune (1998). Artículo: Stakeholder symbiosis. Edición Marzo. Estados Unidos.

Francés, Antonio (2001). Estrategia para la Empresa en América Latina. Caracas, Venezuela: Ediciones IESA.

Francés, Antonio (2003). La Corporación Venezolana y su evolución hacia el siglo XXI. Revista Debates IESA. 2do Trimestre 2003.
Francés, Antonio y Dávalos, Lorenzo. (1992). La Corporación en Cuatro Dimensiones. Caracas, Venezuela: Ediciones IESA.

Hill, Charles. y Jones, Garett (1996). Administración Estratégica: un enfoque integrado. Bogotá, Colombia. Editorial McGraw Hill Interamericana.

Hodge, Billy. y Gales, Lawrence (1997). Teoría de la Organización: un enfoque estratégico.

Johnson, Gerry y Sholes, Kevan (1999). Dirección Estratégica. 3ra. Edición. España: Editorial Prentice Hall.

Kenneth, Rosen (1980). The concept of Corporate Strategy. EE.UU: Richard Irving, Inc.

Kotler, Philip (1996). Dirección de Mercadotecnia. 8va. Edición. México: Editorial Prentice Hall.

Lira, Tomas (2002). Análisis de Grupos de Interés: Enfoque Metodológico, (Documento Inédito). Venezuela: PDVSA.

Madia De Souza, Francisco (1995). La Sexta Generación del Marketing. Colombia: Editorial McGraw-Hill.

Mintzberg, Henry (1997). EI Proceso Estratégico. México: Editorial Prentice Hall Hispanoamericana.

Perozo de Jiménez, Graciela (2002). Imagen Corporativa de una Universidad a Distancia. Trabajo de doctorado no publicado. Universidad Dr. Rafael Belloso Chacín. Maracaibo, Venezuela.

Pizzolante, Italo (1996). Reingeniería del Pensamiento Caracas, Venezuela. Editorial Panapo.

Pizzolante, Italo (1999 a). La Miopía Corporativa (Manuscrito) (Documento Inédito). Caracas, Venezuela: Centro de Estudios IESA.

Pizzolante, Italo (1999 b). Identidad e Imagen: Estrategia para crecer (Manus- 
crito). (Documento Inédito). Caracas, Venezuela: Centro de Estudios IESA.

Ribes, Paul (2003). La Cultura en las Organizaciones. Programa Ejecutivo 2003. IESA.

Stoner James, Freeman Eduard. y Gilbert Daniel (1996). Administración. 6ta Edición. México. Prentice Hall Hispanoamericana.
Van Riel, Cees (1997). Comunicación Corporativa. España: Editorial Prentice Hall.

Young, David (1996). Building your Company's Good Name. EE.UU: American Management Association. 